\section{Kidney \\ Blood Pressure Research}

\title{
Curcumin Ameliorates Podocytic Adhesive Capacity Damage Under Mechanical Stress By Inhibiting miR-124 Expression
}

\author{
Dong Lia, Zhenyu Lu ${ }^{\mathrm{b}, c, d}$ Junya Jiaa ${ }^{\mathrm{a}}$ Zhenfeng Zheng ${ }^{\mathrm{a}} \quad$ Shan Lin ${ }^{\mathrm{a}}$
}

aDepartment of Nephrology, General Hospital of Tianjin Medical University; bUnion Stem Cell \& Gene Engineering Co., Ltd.; 'Center for Reproductive Medicine, The First Teaching Hospital of Tianjin University of Traditional Chinese Medicine, Tianjin, China; ${ }^{\mathrm{T} T}$ These authors contributed equally to this work

\section{Key Words}

miRNA-124 • Curcumin • Podocyte $・$ Diabetic nephropathy $・$ Mechanical stress

\begin{abstract}
Background/Aims: Curcumin, a kind of plant polyphenolic compound, has been recently discovered to have renoprotective effects on diabetic nephropathy (DN). Podocyte can respond to various injuries including mechanical stress secondary to DN. Our previous study showed that podocyte miR-124 expression was up-regulated accompanied with podocytic adhesive capacity damage in vitro and in vivo. We hypothesized, in the present research that curcumin would ameliorate podocyte adhesion damage under mechanical stress by inhibiting miR-124 expression. Methods: Gene expression of miR-124 was measured by real-time PCR and protein expression of integrin $\alpha 3$ was measured by Western blotting in STZ-induced uninephrectomized diabetic rats and cultured podocytes under mechanical stress treated with curcumin respectively. Western blot and luciferase reporter assays were used to detect the effects of miR-124 overexpression on the Itga3 expression in podocytes. Results: Gene expression of miR-124 was upregulated and $\alpha 3$ was downregulated in renal cortex of diabetic rats and cultured podocytes under mechanical stress which were ameliorated by curcumin treatment significantly. Transient co-transfection of miR-124 mimics with luciferase expression plasmids resulted in a significant repression of luciferase activity in podocytes. Mechanistically, Itga3 may be a regulation target of miR-124. Conclusions: These results provide a novel idea that curcumin prevents against podocytic adhesive capacity damage under mechanical stress by inhibitting miR-124.
\end{abstract}

Copyright $@ 2014$ S. Karger AG, Basel

\section{Introduction}

Diabetic nephropathy (DN) is a leading cause of end-stage renal disease in patients and affects approximately $40 \%$ of type I and type II diabetic patients [1]. Multiple 


\section{Kidney Blood Pressure Research}

Kidney Blood Press Res 2013;38:61-71

DOI: 10.1159/000355755
Published onIne: February 18, 2014

(C) 2014 S. Karger AG, Basel

www.karger.com/kbr

Li/Lu/Jia/Zheng/Lin: Curcumin and Podocytic Adhesive Capacity Damage

pathogenic factors are related to the development and progression of $\mathrm{DN}$, for example hemodynamic stimuli, production and release of cytokines, chemokines and growth factors et al [2]. Hemodynamic changes, characterized by increases in high intracapsular pressure, hyperfusion and hyperfiltration are central to the initiation of DN and one of the important pathogenesis of DN [3]. High glomerular capillary pressure will lead mechanical stress to all of the glomerular components and hence structural abnormalities, even glomerulosclerosis [2]. Podocytes are distributed along the glomerular basement membrane to form the outer exocapillary layer of the glomerular filtration barrier and podocyte injury is a major contributor to the pathogenesis of DN $[4,5]$. Mechanical stress may provide deleterious adhesive effects on podocyte due to glomerular capillary hypertension even in early stage of DN [6].

Angiotensin converting enzyme inhibitors (ACEI) and angiotensin II receptor blockers (ARB) have been proposed for the standard clinical treatment of DN. But misgivings remain about their safety and side-effects, such as hyperkalemia, acute renal failure(ARF), et al especially to those patients whose glomerular filtration rate begins to fall. Therefore, there is increasing demand for natural antidiabetic medicines that do not have the adverse effects of modern drugs. Curcumin, is the main active component of turmeric isolated from the plant Curcuma longa $\mathrm{L}$ and has been extensively demonstrated as a functional molecule mediating multiple targets implicated in DN $[7,8]$. It has been reported that curcumin has no severe toxicity, except for minor gastrointestinal adverse effects, even up to the dose of $4 \mathrm{~g}$ for 1 month [9]. The effect of curcumin in preventing the complications of DM, including DN is attributed to its features of antioxidative, anti-inflammatory, hypoglycemic and hypolipidemic activities [10]. However, to the best of our knowledge, studies have not been revealed the effect of curcumin on Hemodynamic changes in DN. We hypothesized, in the present research that curcumin would ameliorate hemodynamic stimuli to glomerulus which is considered to be initial factor of albuminuria in DN [3].

MicroRNAs (miRNAs) are a group of endogenous, noncoding small RNAs that have recently been implicated in the regulation of gene expression on the post-transcriptional level in multiple biologic processes [11]. Recent evidence suggested that miRNAs contributed greatly to podocyte damage which provided us critical new information to advance the knowledge of DN [12-14]. In vitro study of podocyte, we found that miR-124 was upregulated under mechanical stress and might play an important role in the podocytic adhesion damage [15]. The purpose of this study is to investigate the effect of curcumin on podocyte injury under mechanical stress and whether miR-124 is involved which would further explore curcumin's potential mechanism against DN.

\section{Materials and Methods}

\section{Animals}

A total of 35 male Wistar rats (180-220 g body weight; 4 to 6 weeks of age) were purchased from Center of Experimental Animal of Institute of Radiation Medicine of Peking Union Medical College. 25 rats were anaesthetized by intraperitoneal injection with sodium pentobarbital $50 \mathrm{mg} / \mathrm{kg}$ body weight, and the left kidney was removed through a flank incision. We also prepared sham-operated rats, which underwent a similar flank incision followed by kidney exteriorization only (Group CTL, $n=10$ ). Following the operation, uninephrectomized rats were injected with streptozotocin (STZ, $65 \mathrm{mg} / \mathrm{kg}$, Sigma, St. Louis, MO, USA) dissolved in $0.1 \mathrm{~mol} / \mathrm{L}$ sodium citrate buffer ( $\mathrm{pH} 4.0$ ) via a tail vein to induce diabetes. No rats were excluded from the study. Uninephrectomized STZ-injected rats considered diabetic 72 hours later after the injection (blood glucose level of $\geq 16.7 \mathrm{mmol} / \mathrm{L}$ ) were randomly divided into two groups. One group was given $200 \mathrm{mg} / \mathrm{kg} / \mathrm{d}$ curcumin (Tokyo Chemical Industry Co., Ltd, Tokyo, Japan) by daily gavage for 2 weeks after STZ administration (the CUR group, $n=12$ ). Another group was given only distilled water instead of curcumin (the DIA group, $\mathrm{n}=13$ ). Body weight was recorded weekly throughout the experimental period. On week 2 , individual rats were placed in metabolic cages for 24-hour urine specimens collection and urinary albumin test. After urine collection, the animals were sacrificed and right kidney was removed, weighted, 


\section{Kidney \\ Blood Pressure Research}

Li/Lu/Jia/Zheng/Lin: Curcumin and Podocytic Adhesive Capacity Damage

macroscopically divided into renal cortex and medulla. Cortical tissue of right kidney was frozen in liquid nitrogen, and kept at $-80^{\circ} \mathrm{C}$ for biochemical analysis. Partial cortical tissue of right kidney was perfused and fixed with $10 \%$ neutral-buffered formalin for immunofluorescence. All procedures were carried out in accordance with the approval of the ethics committee of Tianjin Medical University.

\section{Cell culture}

A conditionally immortalized human podocyte cell line was kindly donated by Professor Moin Saleem (Bristol Royal Hospital for Children, Bristol, UK). Podocytes clones were cultured in RPMI1640 with 10\% fetal bovine serum (FBS), $100 \mathrm{U} / \mathrm{ml}$ penicillin and $100 \mathrm{mg} / \mathrm{ml}$ streptomycin in the presence of $10 \mathrm{units} / \mathrm{ml}$ interferon- $\gamma$ at $33^{\circ} \mathrm{C}$ in $5 \%$ CO2/95\% air (permissive condition) for propagation. To differentiate, podocytes were plated on type I collagen at a density of $1 \times 10^{4}$ cells $/ \mathrm{cm}^{2}$ and cultured with $1 \% \mathrm{FBS}$ at $37^{\circ} \mathrm{C}$ without $\gamma$-interferon (non-permissive condition). Cells were switched to non-permissive condition for 10-14 days before applying mechanical stress.

\section{Mechanical stretch and curcumin treatment}

Podocytes covered with $2 \mu \mathrm{g} / \mathrm{cm}^{2}$ human extracellular matrix and collagen IV (Santa-Cruz Biotechnology, California, USA) were seeded in six-well Flexplates (Flexcell International Corp., Hillsborough, USA). Stretching was carried out using FX-4000T ${ }^{\text {тм }}$ Cell Stretcher (Flexcell International Corp., Hillsborough, USA) at a frequency of 12 cycles/min and elongation strength of $20 \%$. Certain cells were subjected to mechanical stretch for a period of up to $24 \mathrm{~h}$ (Group M), whereas others were grown in wells with flexible membranes but were not subjected to mechanical stress serving as controls(Group C). To figure out potential interactions between curcumin and mechanical stretch, further experiments were performed on podocytes exposed to mechanical stretch in the presence of curcumin (Group T) (20ug/ml; Tokyo Chemical Industry Co., Ltd, Tokyo, Japan). The concentration of curcumin used in this study was determined in preliminary experiments. A portion of cells and supernatants were collected and stored at $-80{ }^{\circ} \mathrm{C}$ for biochemical analysis. Another portion of cells were then harvested and prepared for microscopy.

\section{Cell adhesion assay}

Cell adhesion was performed as described [16]. Cells $\left(2 \times 10^{5}\right)$ were seeded in triplicates in the sixwell Flexplates. Non-adherent cells were washed off after being exposed to mechanical stress for $24 \mathrm{~h}$ and cells sticking to the bottom were fixed with $4 \%$ paraformaldehyde, stained by $0.1 \%$ crystal violet. The dye was then washed away and the cellular stain was dissolved in 33\% acetic acid. The control cells which grown under identical conditions, but not exposed to stretch were fixed without being washed. Absorbance was quantified with a spectrophotometer at $620 \mathrm{~nm}$ optical density. The percentage of podocyte adhesion is presented as the absorbance of experimental groups divided by the absorbance of the control cells (normalized to 1).

Quantitative reverse transcription-polymerase chain reaction ( $q R T-P C R$ )

Quantification of miR-124 expression levels was assessed via qRT-PCR using specific TaqMan® assays according to the instructions of the manufacturer (Applied Biosystems, Foster City, CA, USA). U6 RNA was used as normalizer.

\section{Immunofluorescence staining}

Frozen kidney sections ( $5 \mu \mathrm{m}$ ) were incubated with bovine serum (5\%) for $30 \mathrm{~min}$ at room temperature. The slides were incubated with rabbit anti-rat integrin $\alpha 3$ monoclonal antibody (Bosider, Wuhan, China) at a 1:200 dilution for overnight at $4^{\circ} \mathrm{C}$. After washing, FITC-labeled goat anti-rat IgG (Invitrogen, Carlsbad, CA, USA) was used as the secondary antibody at a dilution of $1: 100$ at $37^{\circ} \mathrm{C}$ for $90 \mathrm{~min}$.

Podocytes were fixed with $4 \%$ paraformaldehyde for $30 \mathrm{~min}$ at room temperature and then permeabilized with PBS containing $0.2 \%$ Triton X-100 and 2\% BSA for an additional 30 min at room temperature. After blocking, cells were incubated with rabbit polyclonal anti- $\alpha 3$ antibody (Santa Cruz Biotechnology, Santa Cruz, CA, USA) at 1:200 dilution in permeabilization buffer overnight at $4^{\circ} \mathrm{C}$. Antibody binding was detected with secondary anti-rabbit antibody conjugated with FITC at a concentration of 1:200. Nuclei of all cells were counterstained with DAPI. Cells were viewed by the laser-confocal microscope (Leica, Munich, Germany). 


\section{Kidney Blood Pressure Research}

Kidney Blood Press Res 2013;38:61-71

DOI: 10.1159/000355755
Published onIne: February 18, 2014

C 2014 S. Karger AG, Basel

www.karger.com/kbr

Li/Lu/Jia/Zheng/Lin: Curcumin and Podocytic Adhesive Capacity Damage

Western blotting

Renal cortices or podocytes were lysed in hypotonic lysis buffer (Beyotime, Jiangsu, China) with protease/phosphatase inhibitors (1\% phenylmethanesulfonyl fluoride and $1 \%$ cocktail) and then centrifuged at 12,000 rpm for $20 \mathrm{~min}$ at $4^{\circ} \mathrm{C}$. The protein samples were mixed with loading buffer and then heated at $95^{\circ} \mathrm{C}$ to $100^{\circ} \mathrm{C}$ for $5 \mathrm{~min}$. Separated proteins were transferred to a nitrocellulose membrane and blocked with $8 \%$ nonfat milk at room temperature for 1 hour. Membranes with proteins were incubated with rabbit anti-human integrin $\alpha 3$ monoclonal antibody (1:100, Bosider, Wuhan, China) overnight. Horseradish peroxidase-conjugated secondary antibodies (Cell Signaling Technology, Beverly, MA, USA) were used at 1:5,000. Blots were visualized by the enhanced chemiluminescence Western blotting system (Santa Cruz Biotechnology Inc., Santa Cruz, CA, USA) and developed on the film.

\section{DNA construction}

MiR-124 mimics, anti-miR-124, control mimics, Itga3-siRNA and nonsilencing siRNA were synthesized by Shanghai Genepharma Co. Ltd. (Genepharma, Shanghai, China). The primers of full-length Itga3 with target 3' UTR were as follows: Forward, 5'- GCTGGAACTGTCCTAGTCGCGGTTGTCGTC -3'; Reverse, 5'CGCACATGTATTCTCTAGCCTTACGTGCTC-3'. The oligonucleotide (1100 to 1220) of Itga3 3' UTR was synthesized and inserted into pmirGLO luciferase plasmid using PmeI and Xbal restrictions. Putative miR124 binding site GUGCCUU (nt 1160-1166) was mutated into AGUAACG by oligonucleotide-directed PCR (Figure 6B).

\section{Liposome mediated plasmid transfection}

Specific miR-124 mimics, anti-miR-124 molecules, miR-124 mimics negative control, anti-miR-124 negative control, Itga3-siRNA and nonsilencing siRNA were transfected into podocytes at a final concentration of $20 \mathrm{nmol} / \mathrm{L}, 20 \mathrm{nmol} / \mathrm{L}, 20 \mathrm{nmol} / \mathrm{L}, 20 \mathrm{nmol} / \mathrm{L}, 30 \mathrm{nmol} / \mathrm{L}$ and $30 \mathrm{nmol} / \mathrm{L}$ respectively using Lipofectamine 2000 (Invitrogen, Carlsbad, CA, USA). Refer to the Invitrogen manual, 6-hole culture plate was used and added culture medium containing $5 \times 10^{5}$ cells to each hole, at $37^{\circ} \mathrm{C}$ in $5 \% \mathrm{CO} 2$ culture conditions to $50 \%-60 \%$ confluence. After washing with OPTI-MEMI, $1 \mathrm{ml}$ OPTI-MEMI medium was added without FBS. Cells were then added to $200 \mu \mathrm{L}$ plasmid-liposome complexes (plasmid/liposome is $4 \mu \mathrm{g} / 10 \mu \mathrm{g}$ ) to incubate for $5 \mathrm{~h}$. After treatment, they were cultured with $2 \mathrm{ml}$ medium containing $10 \%$ FBS. The transfection efficiency was detected through Real-time PCR or Western blot.

For miRNA targeting luciferase assay, podocytes were co-transfected with $800 \mathrm{ng}$ Luciferase vector, including the 3' UTR of Itga3, miR-124 mimics or miR-124 mimics negative control, anti-miR-124 molecules or anti-miR-124 negative control at a final concentration of $20 \mathrm{nmol} / \mathrm{L}$ by using Lipofectamine 2000 (Invitrogen, Carlsbad, CA, USA). Luciferase assays were performed by using the dual luciferase reporter assay system (Promega, Madison, WI, USA) 24 h after transfection.

Statistical analysis

Statistical analysis was performed using SPSS 16.0 software. Differences were considered significant at $P<0.05$. Data were presented as Mean \pm SEM and were compared by Student $t$ test or ANOVA as appropriate.

\section{Results}

Curcumin influences expression of integrin $\alpha 3$ and miR-124 in renal cortices of STZinduced uninephrectomized rats

Immunofluorescence staining showed that the renal $\alpha 3$ expression was decreased in STZ-induced uninephrectomized rats compared with that in the control rats $(P<0.05)$ and treatment with curcumin significantly increased the intensity of $\alpha 3$ in the diabetic kidney $(P<0.05)$ (Figure1A). This finding was correlated with western blot analysis, in which, $\alpha 3$ expression in renal tissues was significantly suppressed in diabetic rats compared with nondiabetic rats, whereas curcumin enhanced $\alpha 3$ expression in the diabetic rats (Figure 1B1C). Real-time PCR also demonstrated a significant increase of miR-124 expression in the diabetic rats when compared with that in the control rats, which was significantly attenuated by treatment with curcumin, see Figure 2 . 


\section{Kidney Blood Pressure Research}

Fig. 1. Effect of curcumin on expression of integrin $\alpha 3$ in renal cortices of STZ-induced uninephrectomized rats. A: Immunofluorescence detection of renal $\alpha 3$ expression in different groups. Original magnification $\times 400$. B: Western blot for $\alpha 3$ in different groups. $\beta$-actin served as an internal control.

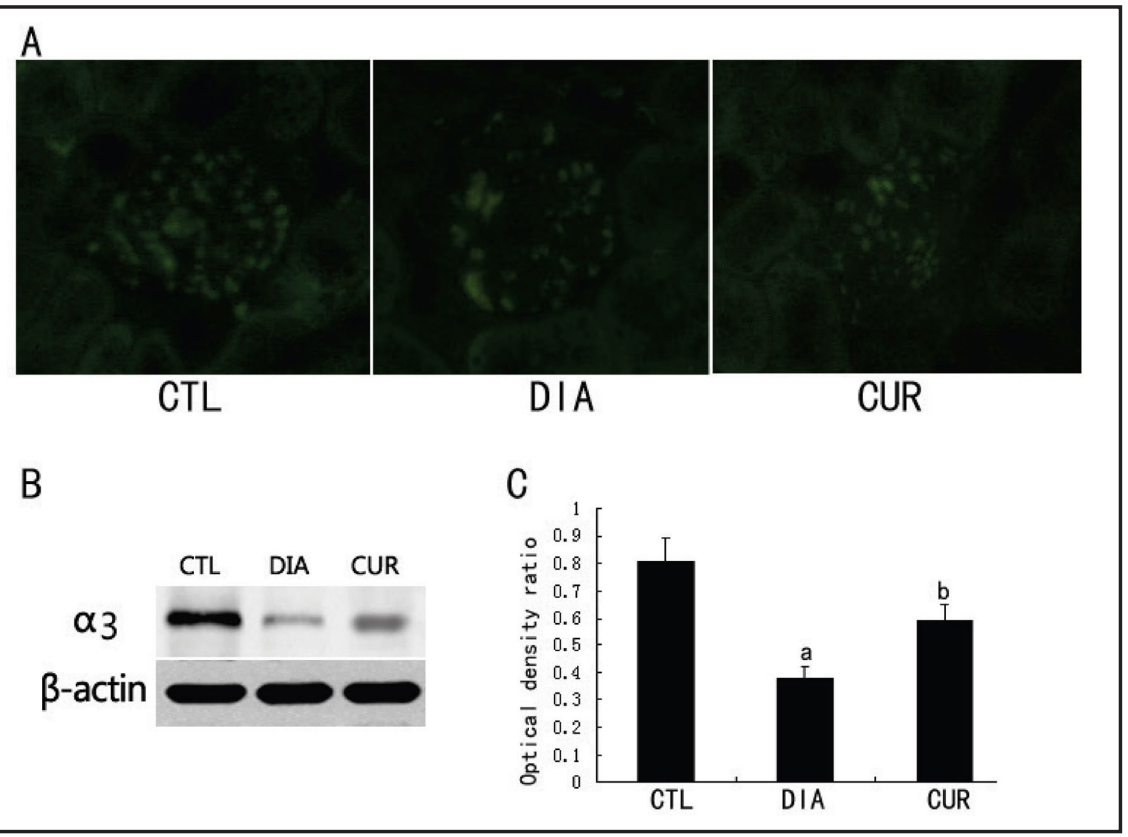
C: The bar graph shows the $\alpha 3$ quantification of each group. ${ }^{a} P<0.05$ vs the CTL group. ${ }^{b} P<0.05$ vs the DIA group $(\mathrm{n}=10-13)$.

Effect of Itga3-siRNA transfection on integrin $\alpha 3$ expression and podocyte adhesion

After Itga3-siRNA transient transfection to podocyte, $\alpha 3$ protein expression and podocyte adhesion capacity were significantly inhibited compared to the control $\operatorname{group}(P<0.05)$ and nonsilencing siRNA group $(P<0.05)$, see Figure 3.

Effect of curcumin on integrin $\alpha 3$ and podocyte adhesion under mechanical stress in vitro

Fluorescent confocal microscopy for integrin $\alpha 3$ confirmed that podocyte $\alpha 3$ expression in Group M was significantly reduced compared with Group C. Curcumin treatment significantly upregulated $\alpha 3$ expression in Group T. Furthermore, cell adhesion under mechanical stress was decreased significantly compared with that of normal podocytes accompanied by downregulation of integrin $\alpha 3$, while curcumin treated group attenuated these changes significantly $(P<0.05)$, see Figure 4. Combined, these findings highlight a critical role for integrin $\alpha 3$ in regulating podocyte adhesion capacity response to mechanical stress

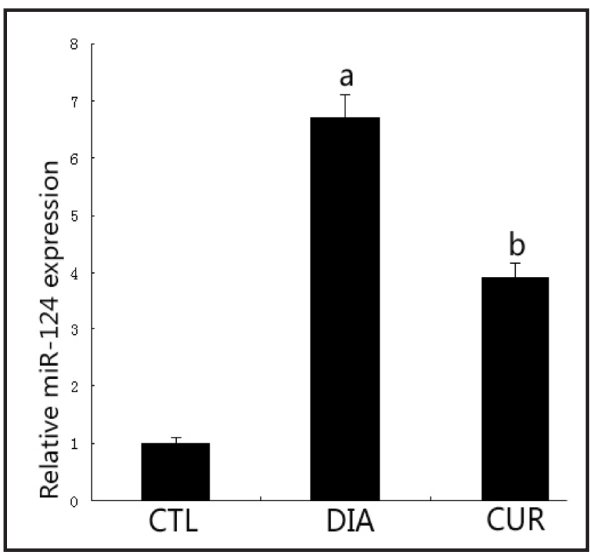

Fig. 2. Changes of miR-124 expression in renal cortices of STZ-induced uninephrectomized rats assessed by Real-time PCR assays. Expression of miR-124 was high in the Group DIA and significantly decreased in Group CUR. ${ }^{a} P<0.05$ vs Group CTL, ${ }^{\mathrm{b}} P<0.05$ vs Group DIA $(\mathrm{n}=10-13)$. in both in vivo and in vitro.

Curcumin influences expression of integrin $\alpha 3$ and miR-124 on podocyte under mechanical stress in vitro

Western Blot analyses showed that podocyte $\alpha 3$ expression was significantly lower in Group M compared to Group C $(P<0.05)$ under mechanical stress. Curcumin significantly upregulated podocyte $\alpha 3$ expression compared to Group M, see Figure 5A-5B. Real-time 


\section{Kidney Blood Pressure Research}

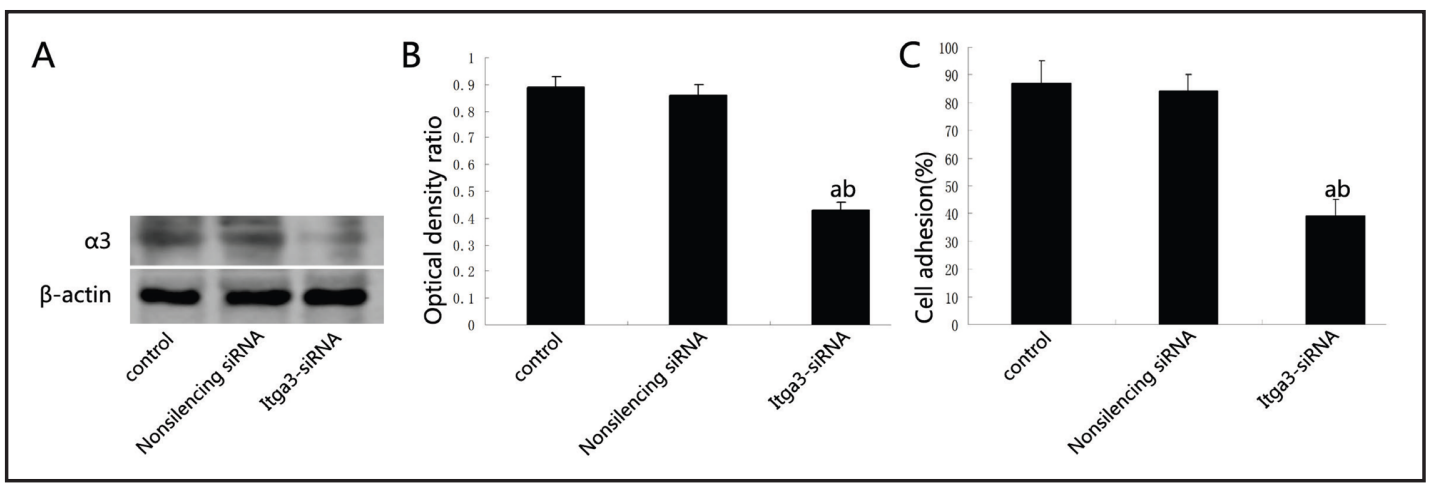

Fig. 3. Effect of Itga3-siRNA transfection on $\alpha 3$ protein expression and adhesion capacity of podocyte. A: Western blot for $\alpha 3$ expression in different groups. $\beta$-actin served as an internal control. B: The bar graph shows the $\alpha 3$ quantification in different groups. C: Podocyte adhesion capacity was decreased significantly after Itga3-siRNA transfection compared to the control group and the nonsilencing group. ${ }^{\text {a }} P<0.05$ vs the control group, ${ }^{\mathrm{b}} \mathrm{P}<0.05$ vs the nonsilencing group $(\mathrm{n}=6)$.

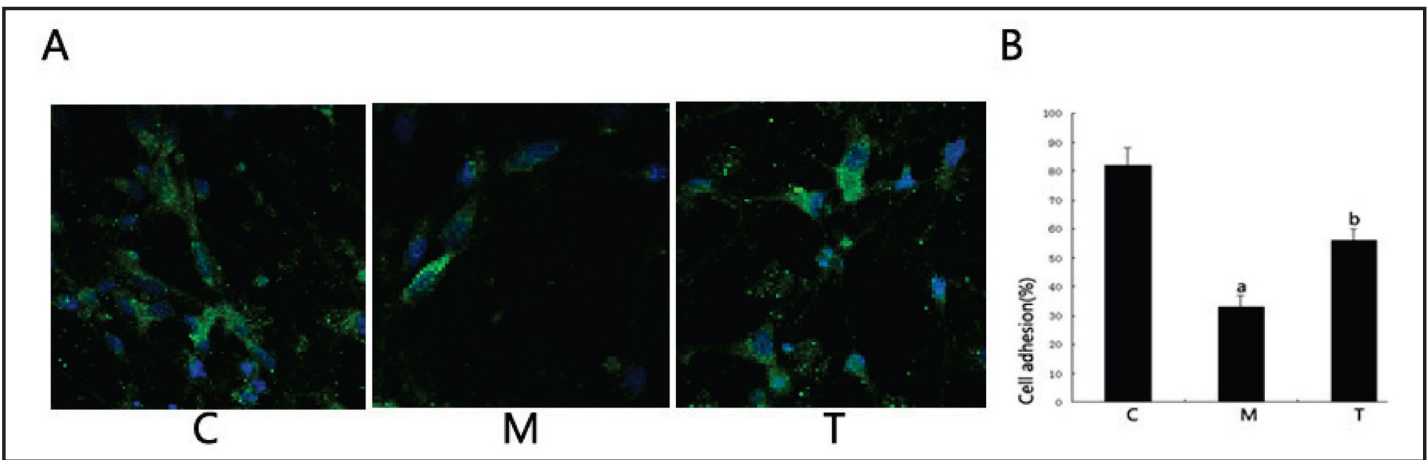

Fig. 4. Effect of curcumin on integrin $\alpha 3$ expression and cell adhesion in each group. A: Immunofluorescence staining shows that integrin $\alpha 3$ is altered profoundly in Group M. Curcumin ameliorates these changes significantly in Group T. B: Podocyte adhesion under mechanical stress was decreased significantly in Group M. Curcumin ameliorates it significantly in Group T. ${ }^{a} P<0.05$ vs Group $C,{ }^{b} P<0.05$ vs the Group M (n=6).

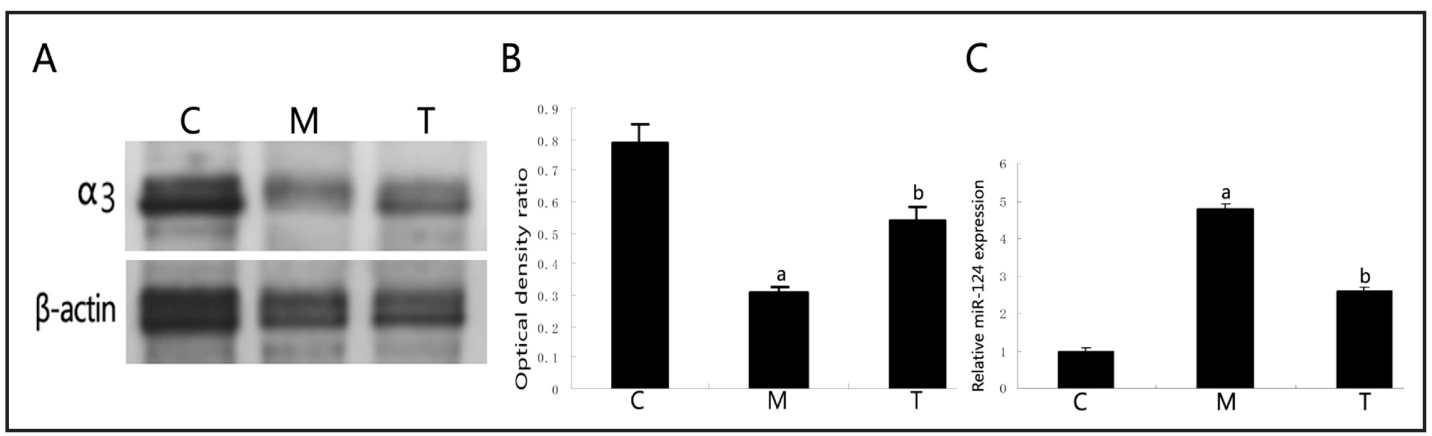

Fig. 5. Effect of curcumin on podocyte expression of integrin $\alpha 3$ and miR-124 under mechanical stress assessed by western blot and Real-time PCR assays. A: Expression changes of integrin $\alpha 3$. $\beta$-actin served as an internal control. B: The bar graph showed the densitometric quantification of $\alpha 3$. C: Expression changes of miR-124. ${ }^{a} P<0.05$ vs Group C, ${ }^{b} P<0.05$ vs the Group M (n=6).

PCR analysis indicated that podocyte miR-124 expression was significantly upregulated in Group M compared with Group C $(P<0.05)$ under mechanical stress and was downregulated significantly after administration of curcumin $(P<0.05)(n=6)$, see Figure 5C. 


\section{Kidney \\ Blood Pressure \\ Research}

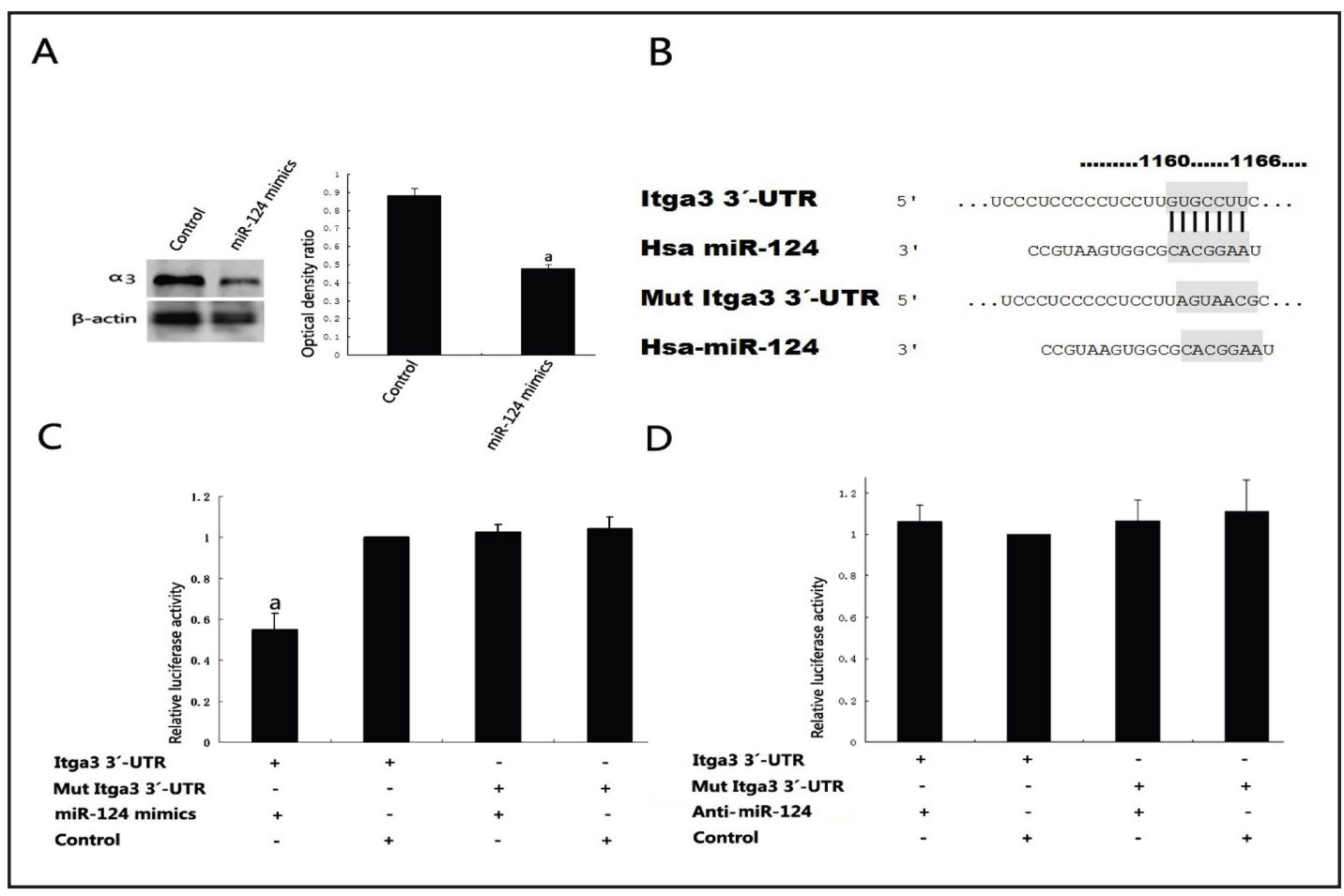

Fig. 6. MiR-124 targets Itga3. A: Podocytes transfected with miR-124 mimics shows decreased integrin $\alpha 3$ expression assessed by Western blot. ${ }^{a} P<0.05$ vs control group. B: A predicted miR-124 target site resides at nt 1160-1166 of the human Itga3 3'-UTR. C: Luciferase reporter assay indicates that miR-124 transfection could decrease luciferase activity in the Itga3 3'UTR group but not in the mutated Itga $33^{\prime}$ UTR group. ${ }^{a} P<0.05$ vs control group. D: Transfection of anti-miR-124 increased luciferase activity and no significant difference was observed. There was also no apparent changes of the luciferase activities when the 3'UTR was mutated $(n=6)$.

\section{MiR-124 targets Itga3}

Bioinformatics database (Targetscan 6.2) and luciferase reporter assay were used to examine the relationship between miR-124 and Itga3. As shown in Figure 6, integrin $\alpha 3$ expression level was downregulated when transfected podocyte with miR-124 mimics compared with the control mimics(Figure 6A). Transient co-transfection of miR-124 mimics with luciferase expression plasmids resulted in a significant repression of luciferase activity in podocytes. However, mutations within putative binding site to Itga3 abrogated the effect of miR-124 mimics (Figure 6C). Furthermore, inhibition of endogenous miR-124 expression using antisense oligonucleotides increased luciferase activity after transfection and no significant difference was observed. There was also no apparent changes of the luciferase activities when the 3'UTR was mutated (Figure 6D). The results above demonstrate that miR-124 directly targets and inhibits Itga3 by binding to its 3'-UTR region.

\section{Curcumin protects podocytic adhesion by inhibiting miR-124 expression}

Because miR-124 promotes podocytic adhesive capacity damage by targeting Itga3, we next used miR-124-overexpressed podocytes to address whether curcumin could protect podocytic adhesive capacity by inhibiting miR-124 expression. Transfection of miR-124 with miR-124 mimics in podocytes resulted in significant cell adhesion decrease compared with those transfected with control mimics, whereas curcumin treatment significantly abrogated these decreases in podocytes accompanied by miR-124 downregulation (Figure 7). 


\section{Kidney Blood Pressure Research}

Fig. 7. Podocytes were transfected with miR-124 mimics and treated with curcumin. Cell adhesion and miR-124 were analyzed by spectrophotometer and Real-time PCR respectively. A: Transfection of miR-124 with miR-124 mimics in podocytes resulted in significant cell adhesion decrease. After curcumin treatment, cell adhesion capacity was improved signi-

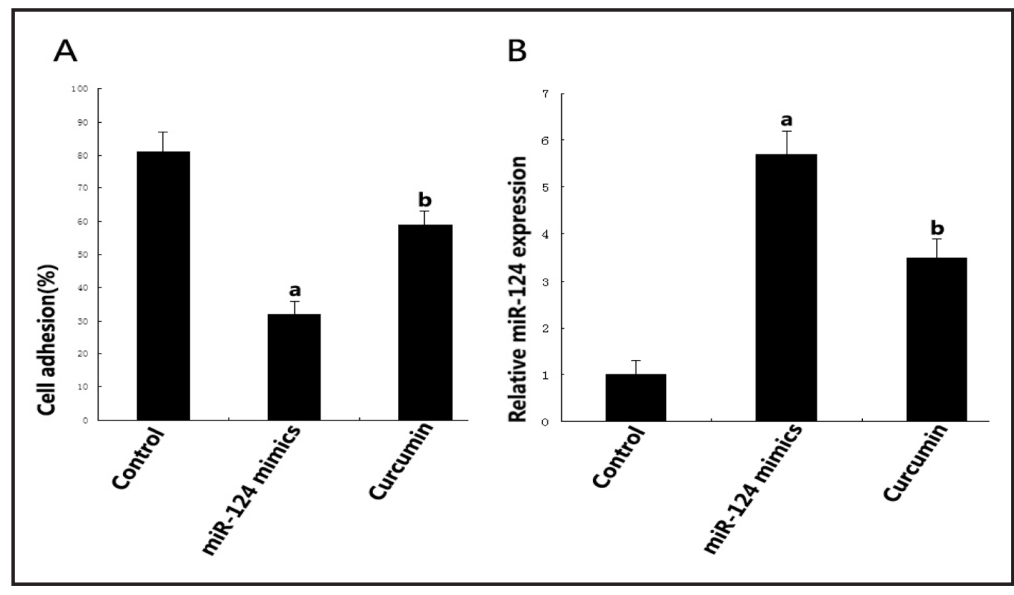
ficantly. B: Podocytes transfected with miR-124 mimics showed significant upregulation of miR-124. Curcumin treatment downregulated miR-124 expression significantly. ${ }^{\mathrm{a}} P<0.05$ vs control group, ${ }^{\mathrm{b}} P<0.05$ vs miR-124 mimics group $(\mathrm{n}=6)$.

\section{Discussion}

Our data show that 1) there exists podocytic adhesive capacity damage in STZ-induced uninephrectomized diabetic rats presented as increased miR-124 expression and decreased integrin $\alpha 3$ expression in renal cortical tissue which was significantly abrogated by curcumin treatment. 2) In vitro, the responsiveness of podocytes to mechanical stress was detected by podocyte adhesion rate, increased miR-124 expression and decreased integrin $\alpha 3$ expression. 3) Itga3 may be a regulation target of miR-124. The results of this study suggest that the benificial effect of curcumin on podocyte in DN is partly mediated by inhibiting miR124 expression.

Hemodynamic changes which are characterised by renal enlargement and hyperfiltration have been shown to correlate with the onset of DN [17]. One of the consequences of changed glomerular capillary hypertension is the increasing mechanical stretch on podocytes which are regarded as intrinsically mechanosensitive cells $[18,19]$. Adhesive capacity damage to podocytes leads to proteinuria, a hallmark of DN. ACEIs or ARBs is recommended as a first-line therapy for patients in early stage of DN, even if they are normotensive. However, their use may be limited by intolerable side effects, such as cough and hypotension, even renal dysfunction and hyperkalemia when intraglomerular pressure decreased. So, new intervention measures with relatively minor side effects on early stage of DN are desperately needed.

Curcumin, a diferuloylmethane, from the golden spice Curcuma longa commonly known as food flavor turmeric, has been recently discovered to have renoprotective effects on DN with minor side effects $[7,20]$. The preventive and therapeutic properties of curcumin are related mainly to its anti-inflammatory and antioxidant properties. It was reported that curcumin treatment protects DN through the inhibition of NF- $\kappa \mathrm{B}$ activity and, as a result, suppresses the expression of proinflammatory cytokines as well as reducing macrophage infiltration in streptozotocin induced-diabetic rats [21]. In addition, curcumin was also found to inhibit lipid peroxidation and augmented the activity of antioxidant enzymes, which in turn attenuated DN [22]. In the present study, our data revealed that there was a significant decrease in expression of cortical integrin $\alpha 3$ in STZ-induced uninephrectomized rats. Administration of curcumin for two weeks significantly up-regulate integrin $\alpha 3$ expression of diabetic rats which may contribute to the protective effects of podocyte adhesion. In vitro study, we proved that podocyte integrin $\alpha 3$ was downregulated under mechanical stress and significantly restored by treatment with curcumin. As we know, this is the first report that 


\section{Kidney Blood Pressure Research}

\begin{tabular}{l|l|l}
\hline Kidney Blood Press Res 2013:38:61-71 \\
\hline DOI: 10.1159/000355755 & (c 2014 S. Karger AG, Basel
\end{tabular}

Published online: February 18, 2014

www.karger.com/kbr

Li/Lu/Jia/Zheng/Lin: Curcumin and Podocytic Adhesive Capacity Damage

podocytic adhesion damage raised by hemodynamic changes could be ameliorated by the administration of curcumin. Because ample evidence indicates that curcumin could regulate multiple molecular targets [10]. The results presented above led to us in-depth analysis and thinking: how does curcumin participate in regulating podocyte integrin $\alpha 3$ under mechanical stress?

A microRNA (miRNA) is defined as a small non-coding RNA of about 22 nucleotides in length that is single-stranded in the functional form. MiRNAs in animals are thought to act primarily as translational repressors by pairing with the 3'-untranslated regions (3'-UTR) of target genes in a complete or in an incomplete complementary manner through its 'seed sequence' in the 5 '-region and controls expression of target genes at the post-transcriptional level $[23,24]$.

Our previous study showed that podocyte miR-124 expression was up-regulated accompanied with podocytic adhesive capacity damage in vitro and in vivo [15, 25]. For exploration of the functional roles of curcumin on podocyte adhesive capacity damage under diabetic conditions, we sought to evaluate potential effects on podocytic miR-124 expression after intervention by curcumin. In our present study, after 24 hours exposure of podocyte to mechanical stress, miR-124 expression was significantly up-regulated accompanied with decreased cell adhesion rate. Treatment with curcumin decreased miR-124 expression markedly and improved podocyte adhesive capacity. Emerging evidence suggests that miRNAs play crucial roles in controlling many cell adhesion molecules thus contribute to normal cell adhesion [26]. A possible mechanism for the precise influence of curcumin on podocyte adhesion capacity under mechanical stress might be through miR-124 targeting Itga3.

Integrins are noncovalently associated, heterodimeric transmembrane proteins which compose of one $\alpha$ and one $\beta$ subunit that are receptors for cell adhesion to extracellular matrix (ECM) [27]. Podocytes are anchored to the GBM principally via $\alpha 3 \beta 1$ integrin whose downregulation is causally related to the podocytic adhesion damage [28]. The integrin $\alpha 3$ knockout mouse exhibits an immature GBM with podocyte foot process effacement and a reduction in the podocyte number [29]. Bioinformatics analyses indicated that there was a highly conserved binding site for miR-124 in the 3'-UTR region of Itga3 in several species. To address whether binding of miR-124 to Itga3 mRNA leads to its translational suppression, we cloned 3'-UTR of the human Itga3 gene into luciferase reporter vector 3.1-luc. Transient cotransfection of miR-124 mimics with luciferase expression plasmids resulted in a significant repression of luciferase reporter gene which suggested that miR-124 directly targeted and inhibitted Itga 3 in podocytes. This may be one of the mechanisms to explain why the pleiotropic effects of curcumin could be in favour of DN.

It's known that one miRNA can pleiotropically regulate the expression of many genes, whereas one gene can also be regulated by multiple miRNAs. MiR-124 which is found to regulate integrin $\alpha 3$ in the present study was also reported to directly regulate integrin $\beta 1$ [30].Our previous study showed that podocyte $\beta 1$ expression was downregulated under mechanical stress accompanied with remarkable increasing of miR-124 [15]. It's highly possible that miR-124 could also target Itgb1 3'-UTR and participate in podocytic adhesive capacity damage under mechanical stress. So, current evidence suggests that miR-124 function as a key modulator in adhesion-associated processes of podocyte. Previous study suggested that mechanical stress to cells could activate NF- $\mathrm{BB}$ transcription factor [31, 32] and curcumin treatment could protect against the development of DN through the inhibition of NF- $\kappa$ B activation [21].

Notably, NF- $\mathrm{KB}$ is an important transcriptional factor that regulates genes which are critical to cell function. It was reported that miRNAs could be transcriptionally regulated by NF- $\kappa B$ [33] As indicated above, in the present study, the potential mechanism how curcumin regulates miR-124 expression during podocytic adhesive capacity damage under mechanical stress might be through inhibiting NF- $\mathrm{KB}$ activation. 


\section{Kidney \\ Blood Pressure Research}

\section{Conclusion}

Our results indicate that curcumin prevents against podocytic adhesive capacity damage under mechanical stress. The effect of curcumin on DN may be mediated by inhibitting miR124 , which regulates the expression level of integrin $\alpha 3$. Given these promising preclinical findings, we believe that the curcumin might be considered as a potential therapeutic option against DN.

\section{Conflict of Interests}

There are no conflicts.

\section{Acknowledgments}

We thank Prof. Moin A. Saleem for providing the podocyte cell line. This work was supported by the National Natural Science Foundation of China, Grant No.81100404.

\section{References}

1 Gross JL, de Azevedo MJ, Silveiro SP, Canani LH, Caramori ML, Zelmanovitz T: Diabetic nephropathy: diagnosis, prevention, and treatment. Diabetes Care 2005;28:164-176.

-2 Giunti S, Barit D, Cooper ME: Mechanisms of diabetic nephropathy: role of hypertension. Hypertension 2006;48:519-526.

-3 Hostetter TH, Rennke HG, Brenner BM: The case for intrarenal hypertension in the initiation and progression of diabetic and other glomerulopathies. Am J Med 1982;72:375-380.

4 Kriz W, Shirato I, Nagata M, LeHir M, Lemley KV: The podocyte's response to stress: the enigma of foot process effacement. Am J Physiol Renal Physiol 2013;304:F333-F347.

-5 Hartner A, Cordasic N, Menendez-Castro C, Volkert G, Yabu JM, Kupraszewicz-Hutzler M, Rascher W, Hilgers KF: Lack of \{alpha\}8-integrin aggravates podocyte injury in experimental diabetic nephropathy. Am J Physiol Renal Physiol 2010;299:F1151-F1157.

-6 Dessapt C, Baradez MO, Hayward A, Dei Cas A, Thomas SM, Viberti G, Gnudi L: Mechanical forces and TGFbeta1 reduce podocyte adhesion through alpha3beta1 integrin downregulation. Nephrol Dial Transplant 2009;24:2645-2655.

7 Huang J, Huang K, Lan T, Xie X, Shen X, Liu P, Huang H: Curcumin ameliorates diabetic nephropathy by inhibiting the activation of the SphK1-S1P signaling pathway. Mol Cell Endocrinol 2013; 365:231-240.

-8 Pan Y, Zhu G, Wang Y, Cai L, Cai Y, Hu J, Li Y, Yan Y, Wang Z, Li X, Wei T, Liang G: Attenuation of high-glucoseinduced inflammatory response by a novel curcumin derivative B06 contributes to its protection from diabetic pathogenic changes in rat kidney and heart. J Nutr Biochem 2013;24:146-155.

-9 Carroll RE, Benya RV, Turgeon DK, Vareed S, Neuman M, Rodriguez L, Kakarala M, Carpenter PM, McLaren C, Meyskens FL Jr, Brenner DE: Phase IIa clinical trial of curcumin for the prevention of colorectal neoplasia. Cancer Prev Res (Phila) 2011;4:354-364.

10 Soetikno V, Suzuki K, Veeraveedu PT, Arumugam S, Lakshmanan AP, Sone H, Watanabe K: Molecular understanding of curcumin in diabetic nephropathy. Drug Discov Today 2013;18:756-763.

11 Pillai RS, Bhattacharyya SN, Filipowicz W: Repression of protein synthesis by miRNAs: How many mechanisms? Trends Cell Biol 2007;17:118-126.

12 Gebeshuber CA, Kornauth C, Dong L, Sierig R, Seibler J, Reiss M, Tauber S, Bilban M, Wang S, Kain R, Böhmig GA, Moeller MJ, Gröne HJ, Englert C, Martinez J, Kerjaschki D: Focal segmental glomerulosclerosis is induced by microRNA-193a and its downregulation of WT1. Nat Med 2013;19:481-487. 


\section{Kidney \\ Blood Pressure Research}

Kidney Blood Press Res 2013;38:61-71

DOI: 10.1159/000355755

(C) 2014 S. Karger AG, Base

www.karger.com/kbr

13 Chen YQ, Wang XX, Yao XM, Zhang DL, Yang XF, Tian SF, Wang NS: MicroRNA-195 promotes apoptosis in mouse podocytes via enhan ced caspase activity driven by BCL2 insufficiency. Am J Nephrol 2011;34:549559.

14 Wang B, Komers R, Carew R, Winbanks CE, Xu B, Herman-Edelstein M, Koh P, Thomas M, Jandeleit-Dahm K, Gregorevic P, Cooper ME, Kantharidis P: Suppression of microRNA-29 expression by TGF- $\beta 1$ promotes collagen expression and renal fibrosis. J Am Soc Nephrol 2012;23:252-265.

15 Li D, Lu Z, Jia J, Zheng Z, Lin S: Changes in microRNAs associated with podocytic adhesion damage under mechanical stress. J Renin Angiotensin Aldosterone Syst 2013;14:97-102.

-16 Yang Y, Guo L, Blattner SM, Mundel P, Kretzler M, Wu C: Formation and phosphorylation of the PINCH-1integrin linked kinase-alpha-parvin complex are important for regulation of renal glomerular podocyte adhesion, architecture, and survival. J Am Soc Nephrol 2005;16:1966-1976.

$\checkmark 17$ Seyer-Hansen K: Renal hypertrophy in experimental diabetes: Some functional aspects. J Diabet Complications 1987;1:7-10.

-18 Ingram AJ, Scholey JW: Stress-responsive signal transduction mechanisms in glomerular cells. Curr Opin Nephrol Hypertens 2000;9:49-55.

19 Endlich N, Kress KR, Reiser J, Uttenweiler D, Kriz W, Mundel P, Endlich K: Podocytes respond to mechanical stress in vitro. J Am Soc Nephrol 2001;12:413-422.

20 Cheng AL, Hsu CH, Lin JK, Hsu MM, Ho YF, Shen TS, Ko JY, Lin JT, Lin BR, Ming-Shiang W, Yu HS, Jee SH, Chen GS, Chen TM, Chen CA, Lai MK, Pu YS, Pan MH, Wang YJ, Tsai CC, Hsieh CY: Phase I clinical trial of curcumin, a chemopreventive agent, in patients with high-risk or pre-malignant lesions. Anticancer Res 2001;21:2895-2900.

-21 Soetikno V, Sari FR, Veeraveedu PT, Thandavarayan RA, Harima M, Sukumaran V, Lakshmanan AP, Suzuki K, Kawachi $\mathrm{H}$, Watanabe K: Curcumin ameliorates macrophage infiltration by inhibiting NF- $\kappa \mathrm{B}$ activation and proinflammatory cytokines in streptozotocin induced-diabetic nephropathy. Nutr Metab (Lond) 2011;8:35.

-22 Soetikno V, Watanabe K, Sari FR, Harima M, Thandavarayan RA, Veeraveedu PT, Arozal W, Sukumaran V, Lakshmanan AP, Arumugam S, Suzuki K: Curcumin attenuates diabetic nephropathy by inhibiting PKC- $\alpha$ and PKC- $\beta 1$ activity in streptozotocin-induced type I diabetic rats. Mol Nutr Food Res 2011;55:1655-1665.

23 Zhao L, Bode AM, Cao Y, Dong Z: Regulatory mechanisms and clinical perspectives of miRNA in tumor radiosensitivity. Carcinogenesis 2012;33:2220-2227.

24 Lai EC: Micro RNAs are complementary to 3'UTR sequence motifs that mediate negative posttranscriptional regulation. Nat Genet 2002;30:363-364.

25 Li D, Lu Z, Jia J, Zheng Z, Lin S: MiR-124 is Related to Podocytic Adhesive Capacity Damage in STZ-Induced Uninephrectomized Diabetic Rats. Kidney Blood Press Res 2013;37:422-431.

26 Valastyan S, Weinberg RA: Roles for microRNAs in the regulation of cell adhesion molecules . J Cell Sci 2011;124: 999-1006

-27 Aberdam D, Galliano MF, Vailly J, Pulkkinen L, Bonifas J, Christiano AM, Tryggvason K, Uitto J, Epstein EH Jr, Ortonne JP, Meneguzzi G: Herlitz's junctional epidermolysis bullosa is genetically linked to mutations in the nicein/kalinin (laminin 5) LAMC2 gene. Nat Genet 1994;6:299-304.

-28 Blattner SM, Kretzler M: Integrin-linked kinase in renal disease: connecting cell-matrix interaction to the cytoskeleton. Curr Opin Nephrol Hypertens 2005;14:404-410.

29 Kreidberg JA, Donovan MJ, Goldstein SL, Rennke H, Shepherd K, Jones RC, Jaenisch R: Alpha3beta1 integrin has a crucial role in kidney and lung organogenesis. Development 1996;122:3537-3547.

30 Cao X, Pfaff SL, Gage FH: A functional study of miR-124 in the developing neural tube. Genes Dev 2007;21:531-536.

-31 Kumar A, Murphy R, Robinson P, Wei L, Boriek AM: Cyclic mechanical strain inhibits skeletal myogenesis through activation of focal adhesion kinase, Rac-1 GTPase, and NF-kappaB transcription factor. FASEB J 2004;18:1524-1535.

32 Zhou H, Li Y, Huang G, Gu X, Zeng J, Li Y, Luo C, Ou B, Zhang Y, Wu Z, Tang L: Interleukin 6 augments mechanical strain-induced C-reactive protein synthesis via the stretch-activated channel-nuclear factor $\kappa \mathrm{B}$ signal pathway. Heart 2013;99:570-576.

-33 Liang M, Yao G, Yin M, Lü M, Tian H, Liu L, Lian J, Huang X, Sun F: Transcriptional cooperation between p53 and NF- $\kappa B$ p65 regulates microRNA-224 transcription in mouse ovarian granulosa cells. Mol Cell Endocrinol 2013;370:119-129. 